ARTICLE

https://doi.org/10.1038/s41467-019-14222-x

\title{
Structure and mechanism of a Type III CRISPR defence DNA nuclease activated by cyclic oligoadenylate
}

Stephen A. McMahon ${ }^{1,3}$, Wenlong Zhu ${ }^{1,3}$, Shirley Graham", Robert Rambo (1) ${ }^{2}$, Malcolm F. White (1) ${ }^{1 \star} \&$ Tracey M. Gloster (1) ${ }^{1 \star}$

The CRISPR system provides adaptive immunity against mobile genetic elements in prokaryotes. On binding invading RNA species, Type III CRISPR systems generate cyclic oligoadenylate ( $\mathrm{COA}$ ) signalling molecules, potentiating a powerful immune response by activating downstream effector proteins, leading to viral clearance, cell dormancy or death. Here we describe the structure and mechanism of a cOA-activated CRISPR defence DNA endonuclease, CRISPR ancillary nuclease 1 (Can1). Can1 has a unique monomeric structure with two CRISPR associated Rossman fold (CARF) domains and two DNA nuclease-like domains. The crystal structure of the enzyme has been captured in the activated state, with a cyclic tetra-adenylate $\left(\mathrm{CA}_{4}\right)$ molecule bound at the core of the protein. $\mathrm{CA}_{4}$ binding reorganises the structure to license a metal-dependent DNA nuclease activity specific for nicking of supercoiled DNA. DNA nicking by Can1 is predicted to slow down viral replication kinetics by leading to the collapse of DNA replication forks.

\footnotetext{
${ }^{1}$ Biomedical Sciences Research Complex, School of Biology, University of St Andrews, North Haugh, St Andrews KY16 9ST, UK. ${ }^{2}$ Diamond Light Source Ltd Diamond House, Harwell Science and Innovation Campus, Fermi Ave, Didcot OX11 ODE, UK. ${ }^{3}$ These authors contributed equally: Stephen A. McMahon, Wenlong Zhu. *email: mfw2@st-andrews.ac.uk; tmg@st-andrews.ac.uk
} 


\section{T} ype III (Csm/Cmr) CRISPR systems utilise a cyclase domain in the Cas10 subunit to generate cyclic oligoadenylate (cOA) by polymerising $\mathrm{ATP}^{1-3}$. The cyclase activity is activated by target RNA binding and switched off by subsequent RNA cleavage and dissociation ${ }^{1,4}$. cOA is a potent antiviral second messenger that sculpts the anti-viral response by binding to and activating CRISPR associated Rossman fold (CARF) family proteins ${ }^{5}$. The only CARF family effectors to be characterised to date are dimeric ribonucleases of the Csx1/Csm6 family, which have a C-terminal HEPN (Higher Eukaryotes and Prokaryotes, Nucleotide binding) domain that is allosterically activated by cOA binding in the CARF domain ${ }^{6-9}$. Csm6 from Enterococcus italicus and Streptococcus thermophilus are activated by cyclic hexa-adenylate $\left(\mathrm{cA}_{6}\right)^{2,3}$, while Csx1 from Sulfolobus solfataricus and Csm6 from Thermus thermophilus are activated by the cyclic tetra-adenylate $\left(\mathrm{cA}_{4}\right)$ molecule ${ }^{1,2,10}$. In addition to ribonucleases, a wide range of CARF family proteins have been identified, including transcription factors such as $\mathrm{Csa} 3^{11}$, $\mathrm{cA}_{4}$-degrading ring nucleases ${ }^{12}$ and putative membrane proteins of unknown function ${ }^{5,13}$, suggesting that much remains to be discovered. The cOA signalling pathway is crucial for defence against mobile genetic elements (viruses and plasmids) in multiple different systems ${ }^{7,14-17}$. In infected cells, activation of Csm6 results in degradation of both host and invading RNA, leading to significant reductions in cell growth until infection is cleared ${ }^{17}$.

T. thermophilus has well characterised type III CRISPR systems and accessory proteins. The T. thermophilus Cmr (type III-B) complex was one of the first studied biochemically, revealing the distinctive cleavage of target RNA with 6 nucleotide spacing ${ }^{18,19}$. High resolution cryo-EM studies revealed the mechanistic basis of this RNA cleavage by the backbone subunit $\mathrm{Cmr} 4^{20}$. The $T$. thermophilus Csm (Type III-A) complex was shown to possess both sequence specific RNA cleavage and RNA-dependent DNA degradation activities, fitting the emerging consensus for type III CRISPR defence ${ }^{21}$. The crystal structure of the T. thermophilus Csm6 (TTHB152) protein revealed a dimeric arrangement with a HEPN nuclease domain possessing weak ribonuclease activity and a CARF domain postulated to bind activating ligands ${ }^{6}$. This was subsequently confirmed with the discovery that the cyclase domain of Cas10 generates cyclic oligoadenylates that activate CARF domain proteins, including Csm6, which is stimulated by $\mathrm{CA}_{4}$ binding to become a much more active ribonuclease ${ }^{2}$. A second Csm6 orthologue (TTHB144) was recently shown to be activated by $\mathrm{cA}_{4}$ binding and also capable of $\mathrm{cA}_{4}$ degradation-the first example of a selflimiting Csm6-family protein ${ }^{10}$.

TTHB155, hereafter Can1 (CRISPR ancillary nuclease 1), was identified as a potential accessory protein of the type III CRISPR system by Shah et al. in 2018 (cluster 107) ${ }^{22}$. The gene is strongly up-regulated following phage infection in T. thermophilus and is situated adjacent to the CRISPR-5 locus, with operons encoding subunits of the Cmr and Csm complexes nearby ${ }^{23}$. Can 1 is a large protein, 636 amino acids in length. Sequence analysis using HHPRED $^{24}$ suggested the presence of two CARF domains, one at the $\mathrm{N}$-terminus and one towards the centre of the protein, together with a C-terminal PD-D/ExK family metal-dependent nuclease domain (Fig. 1a). The protein therefore has a number of unique features that have not been investigated previously. Here, we show that Can 1 is a metal dependent DNA nuclease, activated by $\mathrm{cA}_{4}$ binding. The structure of Can 1 bound to $\mathrm{cA}_{4}$ reveals extensive structural reorganisation around the activator, bringing the two nuclease-like domains together and forming a DNA binding interface with an active site for DNA cleavage. The enzyme preferentially nicks supercoiled DNA to generate ligatable products, which may slow down viral replication without causing catastrophic damage to the host genome.

\section{Results}

Overall structure of Can1 bound to $\mathbf{c A}_{4}$. A synthetic gene encoding Can1 was constructed and cloned into the E. coli expression vector pEV5HisTEV ${ }^{25}$. The protein was expressed with an N-terminal, TEV protease-cleavable polyhistidine tag, and purified by immobilised metal affinity chromatography and gel filtration, with concomitant tag removal, as described in the Methods. Given the observation that the T. thermophilus Csm6 ribonucleases are activated by $\mathrm{cA}_{4}^{2,10}$, we reasoned that Can 1 might bind the same activator. Can1, with selenomethionine incorporated, was crystallised in the presence of $\mathrm{cA}_{4}$. Diffraction data were collected at Diamond Light Source to $1.83 \AA$ resolution and phased using the incorporated selenium atoms. Following autobuilding of the model, it was refined to a final $\mathrm{R}_{\text {work }}$ of $18 \%$ and $\mathrm{R}_{\text {free }}$ of $21 \%$. The protein chain is traced from residue 3 to 638, with three short sections too disordered to model (464-471, 491-494 and 530-539). Efforts were made to crystallise apo Can1, but no crystals were forthcoming.

The structure of Can1 is unique, with four distinct domains linked by flexible loops (Fig. 1). At the N-terminus there is a CARF domain (residues 3-151), domain 2 (residues 168-276), a second CARF domain (residues 293-422) and a nuclease domain (residues 426-638). The two CARF domains display a canonical Rossman fold, with the addition of a $\beta$-strand compared to other reported CARF domain structures; each domain comprises a core of five parallel $\beta$-strands alternating with four $\alpha$-helices (Supplementary Fig. 1). This is followed by a further two $\beta$-strands that form a beta-hairpin, and an $\alpha$-helix. The nuclease domain at the C-terminus comprises a central core of six $\beta$-strands flanked by six $\alpha$-helices typical of the PD-D/ExK metaldependent nuclease family ${ }^{26}$. Domain 2 comprises five $\beta$-strands flanked by four $\alpha$-helices, reminiscent of part of the core domain of the PD-D/ExK nuclease family, with a helix-turn-helix motif between the second and third helices.

$\mathbf{c A}_{4}$ recognition by Can1. There is unambiguous electron density in the $F_{\text {obs }}-F_{\text {calc }}$ map at $3 \sigma$ corresponding to a molecule of $\mathrm{cA}_{4}$ bound to Can1 (Fig. 2a), which was modelled following generation of the library in Acedrg 27 . The molecule of $\mathrm{cA}_{4}$ sits at the interface between the two CARF domains of Can 1 and is completely enclosed by the protein (Fig. 1c). There are a fairly modest number of hydrogen bond interactions between Can1 and $\mathrm{cA}_{4}$ given its size, all but one of which originate from the two CARF domains (Fig. 2b and Supplementary Fig. 2). For clarity, the AMP moieties in $\mathrm{CA}_{4}$ have been arbitrarily named A1-4. There are hydrogen bonds formed between three of the AMP moieties in $\mathrm{CA}_{4}(\mathrm{~A} 1, \mathrm{~A} 2$ and $\mathrm{A} 3$ ) and residues in the first CARF domain; these interactions are formed via main chain atoms of residues Lys90, Asn12, Asp13 and Gly88, and side chain atoms of residues Lys90, Asn12, Tyr143, His113 and Tyr147. Likewise, there are hydrogen bond interactions formed between three of the AMP moieties in $\mathrm{CA}_{4}(\mathrm{~A} 1, \mathrm{~A} 3$ and $\mathrm{A} 4)$ and residues in the second CARF domain; these are formed via main chain atoms of residues Thr384, Gln301, Gly405 and Asn380, and side chain atoms of residues Tyr402, Thr384, Glu324, Thr322, Ser299 and Asn380. A total of 19 water molecules also hydrogen bond directly with $\mathrm{cA}_{4}$.

Superposition of the four adenosine units (centred on the carbon atoms in the ribose sugar) within $\mathrm{cA}_{4}$ show a marked difference in the conformation of one of them (Supplementary Fig. 3). Whilst three of the adenosine moieties superimpose well (with respect to the ribose sugar and imidazole moiety of the adenine base; one adenine base is flipped compared to the other two but in the same plane), the adenine of the other adenosine moiety (A1) is in a more axial position at the anomeric carbon. This adenine base displays a clear $\pi-\pi$ stacking interaction with 
a

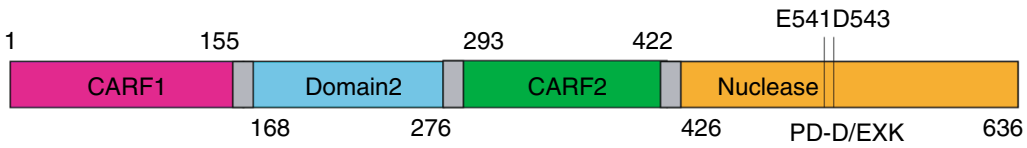

b

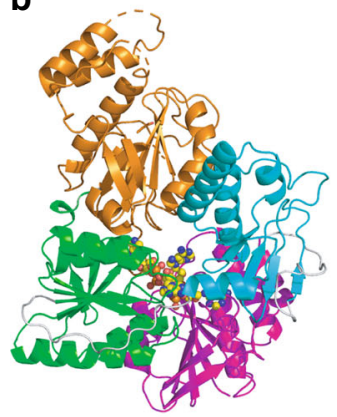

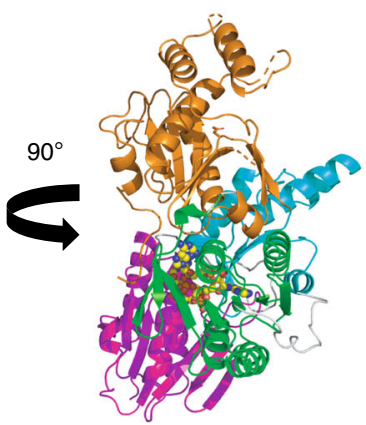

C

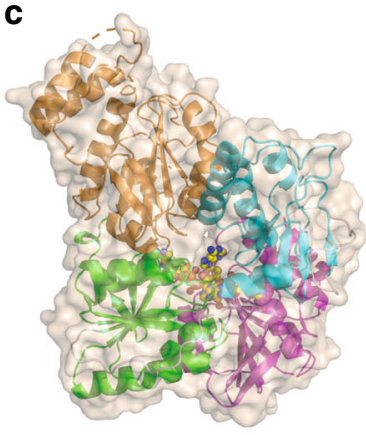

Fig. 1 Domain organisation and structure of Can1. a Domain organisation of Can1 with residue numbers indicated. Residues Glu541 and Asp543 are in the nuclease active site. $\mathbf{b}$ Two views of the overall structure of Can1 shown in cartoon representation. Coloured as in panel (a): first CARF domain (magenta), domain 2/nuclease-like domain (cyan), second CARF domain (green), nuclease domain (orange), loops between domains (grey). A molecule of cA 4 bound to Can1 is shown in sphere representation (carbon in yellow, nitrogen in blue, oxygen in red). c Overall structure of Can1, as coloured in panel (b), with surface representation to illustrate $\mathrm{CA}_{4}$ is completely buried.

Trp42, which appears to be the driving force for this alternate, and most probably higher energy, conformation for the adenine. It has the knock-on effect of causing the ribose sugar to display a 2'-exo conformation, compared to an 2'-endo conformation which is observed in the other adenosine units.

Only two residues from the nuclease domain and domain 2 are in close proximity to $\mathrm{cA}_{4}$. Gly550, from the nuclease domain, forms a hydrogen bond interaction via its main chain carboxyl group to the adenine moiety of A4 (Fig. 2b). Gln222 from domain 2 does not interact directly with any atoms in $\mathrm{CA}_{4}$, but hovers above the centre of the $\mathrm{CA}_{4}$ molecule, and makes two watermediated interactions to opposite phosphate groups in $\mathrm{cA}_{4}$ (Supplementary Fig. 4). To investigate the importance of these interactions for ligand binding, we engineered and purified the CARF domain variants N12A, W42A, K90E, H113A and Q222E. The effects of these changes on $\mathrm{CA}_{4}$ binding were monitored by electrophoretic mobility shift assay (EMSA) using labelled $\mathrm{cA}_{4}$. Binding of $\mathrm{cA}_{4}$ was abolished for the $\mathrm{K} 90 \mathrm{E}$ variant, severely reduced for the N12A variant, and moderately affected for W42A, H113A and Q222E (Supplementary Fig. 5). These observations highlight the distributed nature of $\mathrm{CA}_{4}$ interactions and the crucial role of Lys90, which is situated at the base of the $\mathrm{cA}_{4}$ binding pocket.

All other characterised CARF family proteins consist of a single CARF domain, which homo-dimerises to bind one molecule of cOA. Can1 is unique as it possesses two CARF domains in a single polypeptide. The CARF domains superimpose with an RMSD of $2.5 \AA$ over $113 \mathrm{Ca}$ atoms, although few residues are conserved (Supplementary Figs. 6 and 7A). A striking feature of $\mathrm{CA}_{4}$ recognition is the asymmetry of the interactions formed with Can1, as the interactions with each AMP moiety are distinct. The comparison highlights the structural equivalence of Asp13 and Gln301, which both make main chain interactions with $\mathrm{cA}_{4}(\mathrm{~A} 1$ and $\mathrm{A} 3$, respectively), and of Lys90 and Thr384 (A2 and A4, respectively), which both make main chain and side chain interactions with $\mathrm{cA}_{4}$.

Structural comparisons of the $\mathbf{c A}_{\mathbf{4}}$ binding site. DALI ${ }^{28}$ searches for the nearest structural homologue to each CARF domain in Can1 gave identical results for each: hypothetical protein VC1899 (PDB: 1XMX), a CARF family protein that is a member of the DUF1887 family. The CARF dimer (taken as the two domains from a single polypeptide chain) of Can1 superimposes with the CARF dimer (formed by two monomers) of VC1899 with an RMSD of $3.6 \AA$ over $294 \mathrm{Ca}$ atoms (Fig. 3a). This superimposition allows exploration of likely interactions between VC1899 and $\mathrm{cA}_{4}$. All of the main chain interactions observed between Can 1 and $\mathrm{CA}_{4}$ are likely conserved, as are a number of the side chain interactions (Supplementary Figs. 6 and 7B). There is no equivalent in VC1899 to Trp42, the residue responsible for causing one of the adenine bases (from A1) to sit in a more axial conformation with respect to the ribose sugar. With the absence of this interaction this adenine may not be in such a strained position when bound to VC1899, and there is sufficient room in the binding site for this to fit in a more relaxed position (Fig. 3b). Few close interactions/clashes between $\mathrm{cA}_{4}$ and $\mathrm{VC} 1899$ are predicted, and these involve side chains which could easily be repositioned, suggesting there is little rearrangement of either the CARF dimer or of residues in the CARF domains upon binding of $\mathrm{CA}_{4}$. In other words, with the caveat that $\mathrm{VC} 1899$ has not yet been demonstrated to bind $\mathrm{cA}_{4}$, the $\mathrm{cA}_{4}$ binding site of Can1 is likely to be largely pre-formed in the apo-protein.

The nuclease and nuclease-like domains. DALI searches for structural homologues of the nuclease domain of Can 1 once again gave the hypothetical protein VC1899 (PBD: 1XMX) as the highest hit. The nuclease domain from Can1 superimposes with the nuclease domain of VC1899 with an RMSD of $3.4 \AA$ over 160 $\mathrm{Ca}$ atoms (Fig. 3c, with nuclease active site residues shown). Whilst the core of the nuclease domain is very similar, Can 1 has an extra helix-loop-helix feature between residues 441 and 498, whereas at the equivalent position (residues 156-248) VC1899 possesses a non-superimposable section. The active site residues Glu541, Asp543, Glu560 and Lys562 in Can1 overlap with equivalent active site residues in VC1899. The closest structural homologues with dsDNA bound are the type 2 restriction enzymes AgeI (PDB: 5DWB ${ }^{29}$ ) and NgoMIV (PDB: 1FIU ${ }^{30}$ ), which overlap with the nuclease domain of Can1 with an RMSD of $4.0 \AA$ over $143 \mathrm{Ca}$ atoms and $3.4 \AA$ over $133 \mathrm{Ca}$ atoms, respectively (Supplementary Fig. 8). The core secondary structure elements of the nuclease domain of Can1 and each of the restriction enzymes are conserved, and the active site residue 

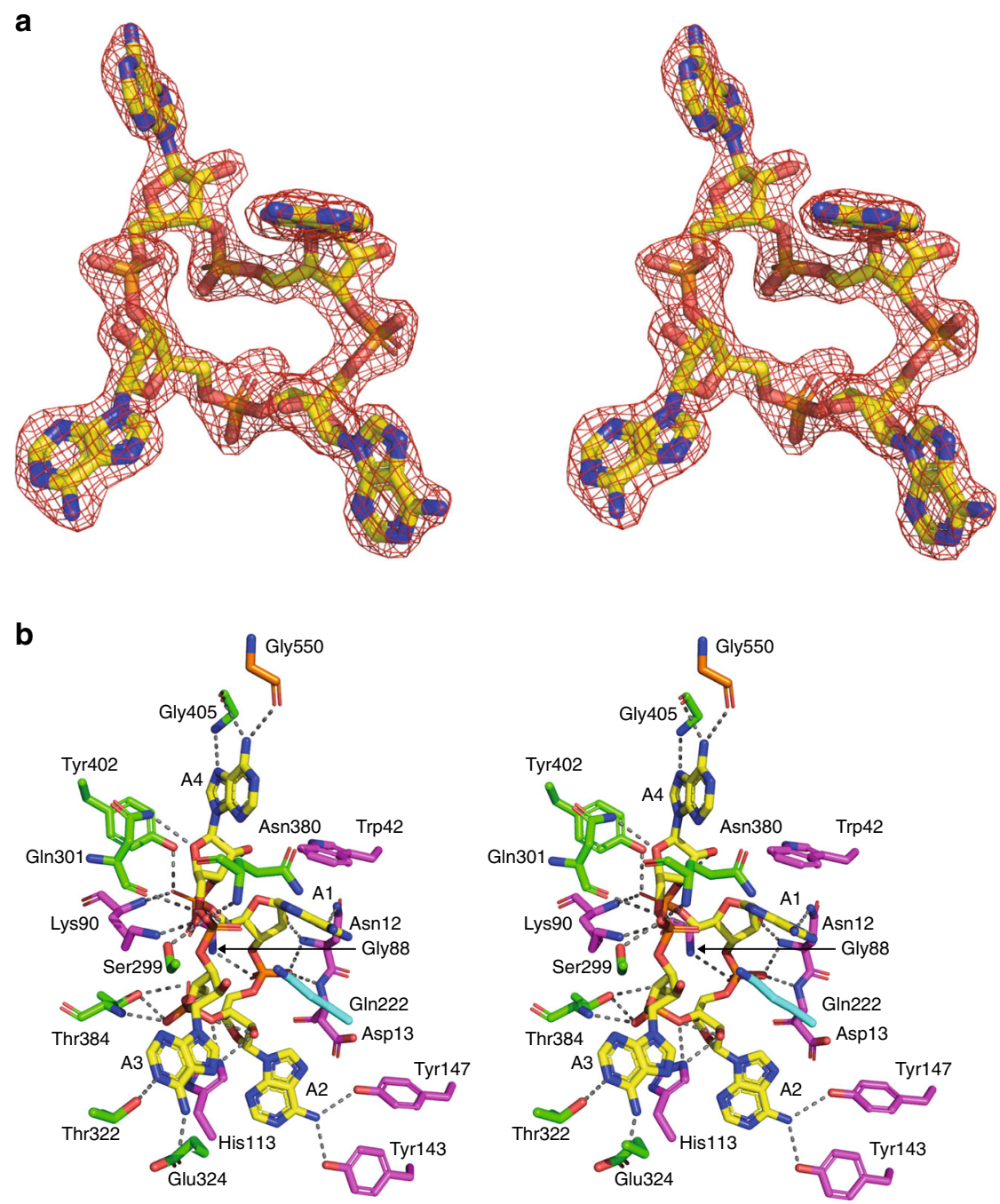

Fig. 2 Structure of the $\mathbf{c A}_{\mathbf{4}}$ activator and interactions with Can1. a Divergent stereo representation of $c A_{4}$ (in stick representation; carbon in yellow, nitrogen in blue, oxygen in red) with the maximum likelihood/ $\sigma_{A}$ weighted $F_{\text {obs }}-F_{\text {calc }}$ electron density map contoured at 3.0 sigma shown in red. b Divergent stereo representation of the binding site of $c A_{4}$ in complex with Can1. $c A_{4}$ (with each AMP numbered A1-A4) and binding site residues are shown in stick representation, with $\mathrm{CA}_{4}$ in yellow and amino acid residues coloured by the domain from which they originate (CARF domain 1: magenta, domain 2/nuclease-like domain: cyan, CARF domain 2: green, nuclease domain: orange). The dotted lines represent hydrogen bond interactions.

a

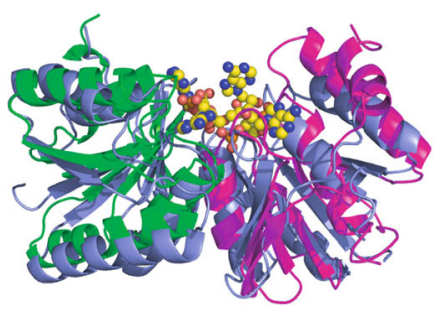

b

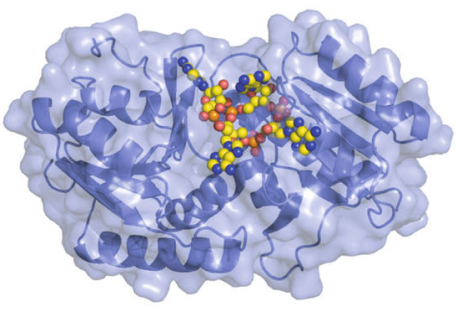

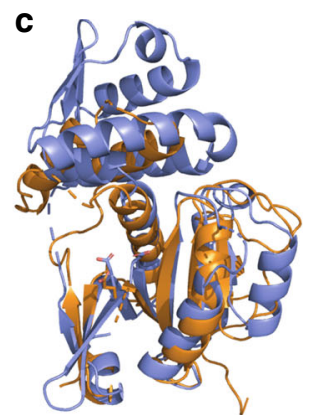

Fig. 3 Structural comparison of Can1 and the DUF1887 family protein VC1899. a Superimposition of the CARF domains from Can1 (coloured as in Fig. 1) in complex with $\mathrm{CA}_{4}$ (yellow spheres) with the CARF domain from VC1899 (mauve). The VC1899 dimer (monomer plus the symmetry mate that forms the functional dimer) was overlapped with Can1. b Surface and cartoon representation of VC1899, with $\mathrm{cA}_{4}$ (yellow spheres) modelled based on the superimposition shown in panel (a). c Superimposition of the nuclease domain from Can1 (orange) and the nuclease domain from VC1899 (mauve). The active site residues are shown as sticks. 


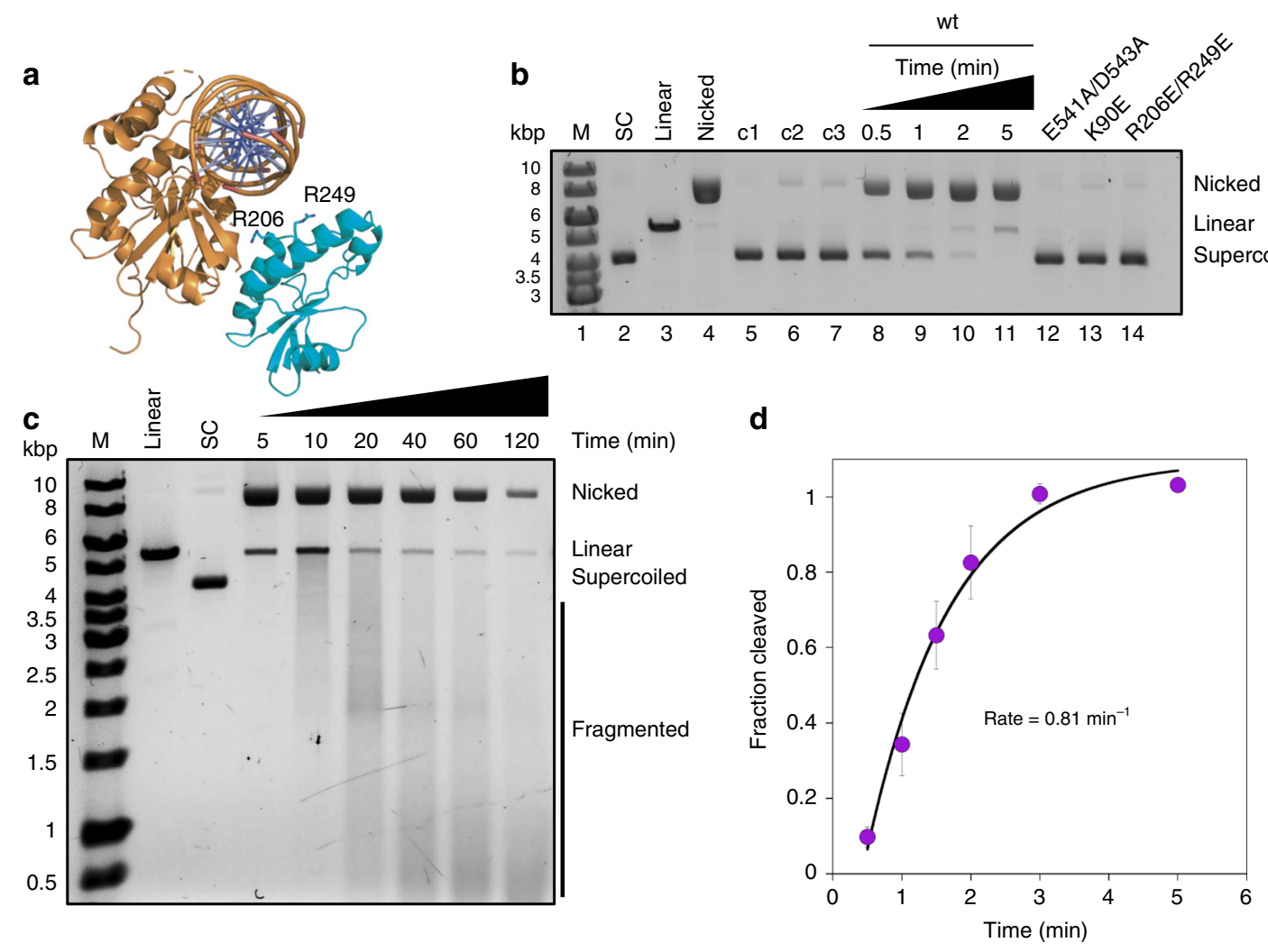

Fig. 4 Can1 nicks supercoiled plasmid when activated by $\mathbf{C A}_{\mathbf{4}}$ in the presence of $\mathbf{M n C l}_{\mathbf{2}}$. a The nuclease domain of Can1 (orange) in complex with the modelled position of dsDNA (based on homologous structures, the nuclease domains of Agel (PDB: 5DWB) and NgoMIV (PDB: 1FIU)). The nuclease-like domain of Can1 is shown in cyan and Arg206 and Arg249 are shown as sticks. b Native agarose gel analysis of plasmid ( 2 nM) cleavage by Can1 (200 $\mathrm{nM}$ ), showing rapid nicking in the presence of $\mathrm{cA}_{4}(200 \mathrm{nM})$ and $\mathrm{MnCl}_{2}(5 \mathrm{mM})$ (lanes 8-11). Standards corresponding to supercoiled, linear and partially nicked plasmid are shown in lanes 2-4. Lanes 5-7 show the reactions incubated for 5 min without protein (c1), $\mathrm{MnCl}_{2}(\mathrm{c} 2)$ or cA 4 (c3), respectively. Can1 variants E541A/D543A, K90E and R206E/R249E generate minimal nicked product after 5 min (lanes 12-14). M-DNA size markers. c Extended time course of plasmid degradation by Can1. Rapid nicking is followed by slower degradation to small linear products over $2 \mathrm{~h}$. Standards are as described in part b. $\mathbf{d}$ Single-turnover kinetic analysis of supercoiled plasmid nicking by Can1, under the conditions in $\mathbf{b}$, yield a rate constant of $0.81 \pm 0.15$ min ${ }^{-1}$. Data points are the mean of triplicate experiments with the standard deviation shown. Source data are provided as a 'Source Data' file.

Asp543 in Can1 overlaps with Asp142 in the AgeI nuclease and Asp140 in the NgoMIV nuclease. Thus, the approximate binding position of the dsDNA in complex with Can1 can be predicted with some confidence (Fig. 4a).

Perhaps more surprisingly, DALI searches revealed that the top hit for homologues of domain 2 of Can1 is also VC1899, which overlaps with an RMSD of $2.5 \AA$ over $86 \mathrm{Ca}$ atoms. Domain 2 of Can1 also superimposes with the nuclease domain of Can 1 with an RMSD of $2.6 \AA$ over Ca 83 atoms, but it lacks the canonical nuclease active site residues of either (Supplementary Fig. 9). We herewith refer to domain 2 as the "nuclease-like domain".

Can1 is a $\mathbf{c A}_{4}$ activated, metal dependent DNA nuclease. The variants E541A/D543A, targeting the $\mathrm{PD}-\mathrm{D} / \mathrm{ExK}$ active site, $\mathrm{K} 90 \mathrm{E}$, targeting a key interaction with $\mathrm{cA}_{4}$ in the CARF domain, and R206E/R249E, targeting a putative DNA binding site in the nuclease-like domain, were expressed and purified as for the wildtype enzyme. Wild-type Can $1(200 \mathrm{nM})$ cleaved a supercoiled plasmid substrate $(2 \mathrm{nM})$ in the presence of $200 \mathrm{nM} \mathrm{cA}_{4}$ and $5 \mathrm{mM} \mathrm{MnCl}_{2}$ (Fig. 4b). The majority of the plasmid was nicked within $1 \mathrm{~min}$, with a small amount linearised after $5 \mathrm{~min}$ incubation. Reactions in the absence of $\mathrm{MnCl}_{2}$ or $\mathrm{cA}_{4}$ showed very little activity, confirming that Can1 is a metal dependent DNA nuclease activated by $\mathrm{CA}_{4}$. Moreover, variants E541A/D543A (nuclease variant) and K90E (CARF domain variant) were both catalytically inactive, as expected for a $\mathrm{cA}_{4}$-activated $\mathrm{PD}$-D/ExK family nuclease. The R206E/R249E variant, which reverses two positive charges on the surface of the nuclease-like domain, was also inactive, suggesting a role for this domain in DNA binding. These residues are in reasonably close proximity to the predicted position of the dsDNA (Fig. 4a), and the nuclease-like domain may move closer still upon DNA binding. Moderate reductions in $\mathrm{cA}_{4}$ cleavage were observed for the other $\mathrm{cA}_{4}$-interacting residue variants tested (Supplementary Fig. 5B).

On extended incubation over $2 \mathrm{~h}$, Can 1 slowly degraded the nicked plasmid to linear fragments of progressively smaller size (Fig. 4c). Thus, the enzyme favours nicking of supercoiled DNA, with more extensive DNA degradation seen only under extended incubation. To measure the rates of supercoiled plasmid nicking under pseudo-single turnover conditions, $200 \mathrm{nM}$ protein was incubated with $2 \mathrm{nM}$ plasmid, and the fraction of supercoiled plasmid cleaved was quantified, normalised, plotted and fitted to an exponential equation as described in the Methods. Two biological replicates and six technical replicates were carried out, yielding a rate of $0.81 \pm 0.15 \mathrm{~min}^{-1}$ (Fig. $4 \mathrm{~d}$ ).

Can1 cleaves supercoiled DNA at random sites. To determine whether Can1 nicks supercoiled DNA at a specific site, we compared its activity to the Nickase Nt.BspQI, which nicks DNA specifically at 5'-GCTCTTCN/ sites, and has one recognition site 


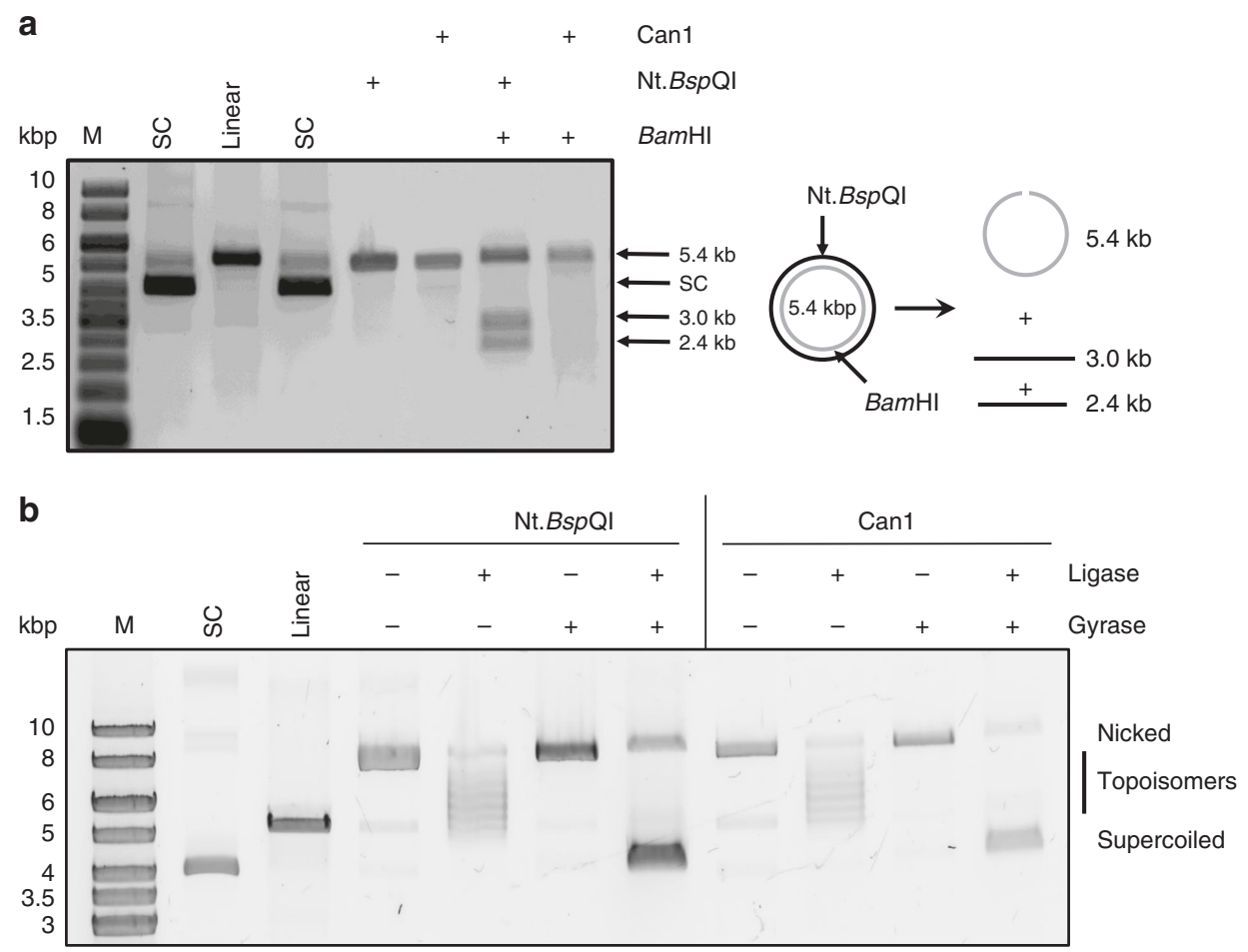

Fig. 5 Can1 nicks plasmid DNA at random sites, leaving ligatable DNA ends. a Plasmid nicking by the Nickase Nt.BspQI and Can1 were analysed by alkaline agarose gel electrophoresis where all DNA is denatured into single-stranded products. Both generate linear $5.5 \mathrm{~kb}$ products. When Nt.BspQI nicking at its recognition site is followed by BamHI digestion, the expected linear products of 3.0 and $2.5 \mathrm{~kb}$ are also observed. Can1, which nicks at random sites, generates a wide range of linear products following BamHI digestion that appear as a smear in the gel. $\mathbf{b}$ Both the Nickase Nt.BspQI and Can1 nick supercoiled plasmid to yield open circle DNA that can be visualised following agarose gel electrophoresis. Ligation of these products by DNA ligase generates closed circular DNA with a range of topoisomers. Addition of DNA gyrase restores the supercoiled DNA substrate. Control lanes are as for Fig. 4. Source data are provided as a 'Source Data' file.

in the plasmid pEV5HisTEV. By using alkaline agarose gel electrophoresis to analyse the cleavage products, we ensured that DNA migrated in single stranded form. Nt.BspQI nicking generated a single product band of $5.4 \mathrm{~kb}$, as expected (Fig. 5a), whilst cleavage by Nt.BspQI followed by BamHI (which also has a single recognition site in pEV5HisTEV) yielded 3 bands-a $5.4 \mathrm{~kb}$ band representing the strand that is cut only by $B a m \mathrm{HI}$, and two smaller bands of 3.0 and $2.4 \mathrm{~kb}$ that result from cleavage of the Nt.BspQI nicked strand by BamHI, as shown in the schematic. Can 1 also generated a nicked strand of $5.4 \mathrm{~kb}$, but when this product was further digested by $B a m \mathrm{HI}$ a smear of smaller DNA products were observed rather than specific fragments (Fig. 5a). This demonstrates that Can1 nicks supercoiled DNA at random sites.

Nucleases within the superfamily which contains the PD-D/ ExK motif typically catalyse phosphodiester bond cleavage by a two metal ion catalytic mechanism ${ }^{26}$. A water molecule is deprotonated by one metal ion and used as a nucleophile to attack the scissile phosphate bond, generating 5'-phosphate and 3'-hydroxyl products ${ }^{26}$. To determine whether Can 1 conformed to this mechanism, we incubated the nicked plasmid generated by Can1 with DNA ligase, followed by DNA gyrase, as described in the Methods. Commercial nicking endonuclease Nt.BspQI was used as a positive control. After incubating nicked plasmid with ligase, various relaxed topoisomers were observed (Fig. 5b), suggesting that the nicked plasmid was ligated to covalentlyclosed circular plasmid. The products were further incubated with DNA gyrase to introduce negative supercoils (Fig. 5b), confirming that ligation of the nicked sites had occurred. Thus, Can1 hydrolyses phosphodiester bonds in supercoiled DNA substrates, generating ligatable 5'-phosphate and 3'-hydroxyl products.
Structural rearrangements upon $\mathbf{c A}_{4}$ binding. The binding of a ligand as large as $\mathrm{CA}_{4}$ will inevitably require structural rearrangements of Can1, particularly as it is completely buried in the complex. As we lacked a crystal structure of apo Can1, we investigated the solution state of apo Can1 and Can1 bound to $\mathrm{cA}_{4}$ using small angle X-ray scattering (SAXS). Size exclusion chromatography coupled SAXS was performed on apo Can1 to ensure optimal background subtraction and sample characterisation, and a single, well defined elution peak was observed. The SAXS derived radius-of-gyration, $\mathrm{R}_{\mathrm{g}}$, shows the apo state is less compact than Can1 in complex with $\mathrm{CA}_{4}$, as characterised by a larger $R_{g}(28.44 \pm 0.13 \AA$ vs $30.13 \pm 0.15 \AA)$, particle volume and maximum dimension, $\mathrm{d}_{\max }$. The dimensionless Kratky plot (Fig. 6a) suggests Can 1 is not globular in either the bound or apo state, which is consistent with the observations of the X-ray crystal structure of Can 1 bound to $\mathrm{CA}_{4}$. Furthermore, analysis of the real-space pairwise distances within the particles, $\mathrm{P}(\mathrm{r})$-distribution, shows the apo state of Can1 has a wider overall distribution (Fig. 6b) with a substantially larger maximum dimension, $\mathrm{d}_{\max }$ (121.5 $\AA$ vs $101.5 \AA$ for the complex), suggesting there is a significant conformational difference between the bound and apo states in solution. In fact, fitting the Can 1 crystal structure to the SAXS data results in a $\mathrm{Chi}^{2}>80$.

To investigate the structural changes required to explain the SAXS dataset of the apo state, we performed a distance restrained, rigid body molecular dynamics simulation that restricted the search to the distances within the apo state $\mathrm{P}(\mathrm{r})$-distribution. The Can 1 crystal structure demonstrated clearly defined domains that were used to define rigid bodies to which centre-of-mass restraints were assigned. Given the observed conservation of the CARF dimer conformation in the $\mathrm{cA}_{4}$ bound Canl and apo 

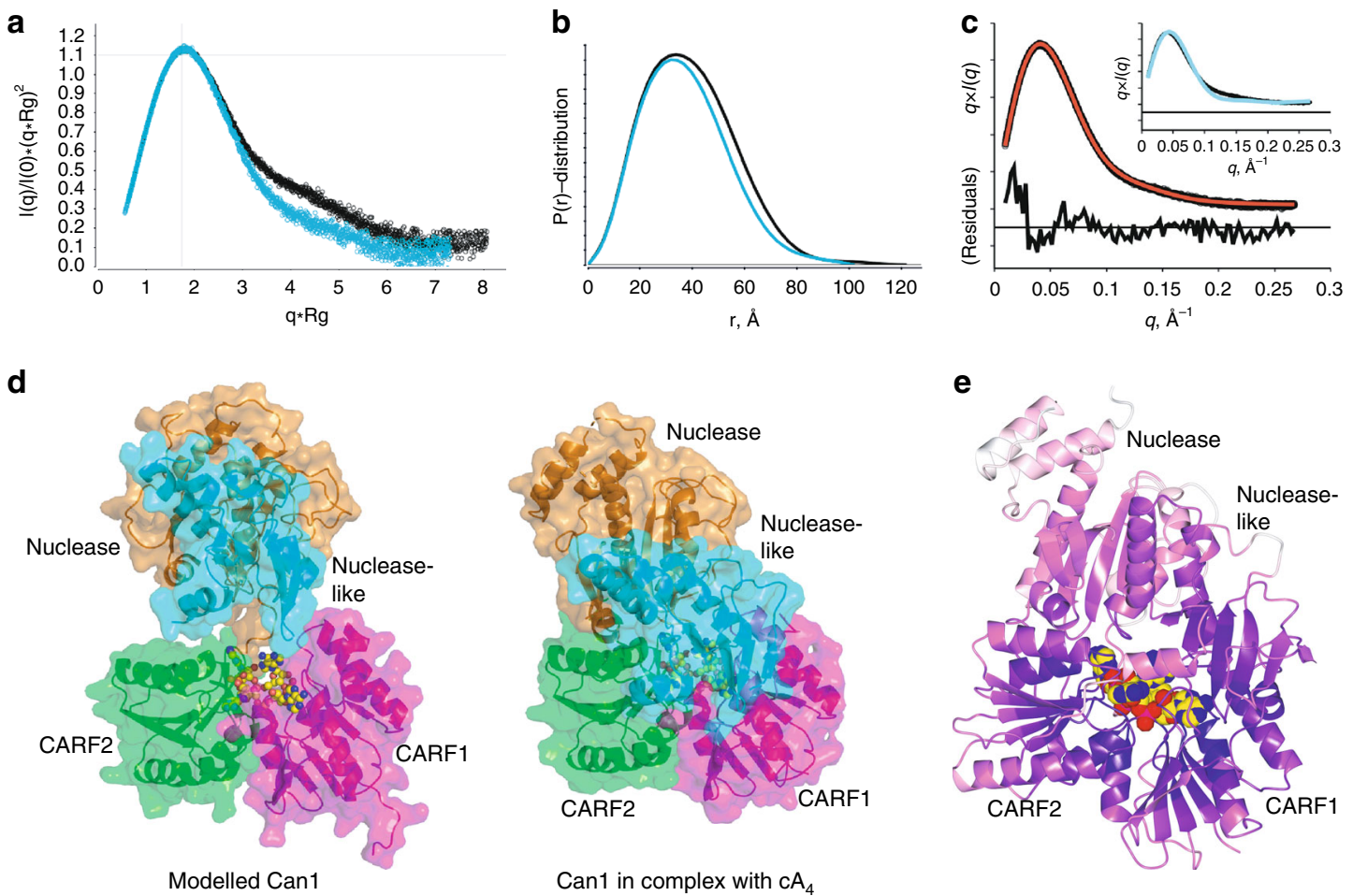

Fig. 6 Structural rearrangements of Can1 upon $\mathbf{C A}_{\mathbf{4}}$ binding in solution. a Dimensionless Kratky plot of Can1 in the presence (blue) and absence (black) of $\mathrm{cA}_{4}$. Data were normalised with $\mathrm{R}_{\mathrm{g}}$ and I(0) of $28.88 \pm 0.13 \AA$ and $1.090 \mathrm{E}-1 \pm 1 \mathrm{E}-4$ respectively for Can 1 with $\mathrm{cA} \mathrm{A}_{4}$ and $30.26 \pm 0.11 \AA$ and $8.99 \mathrm{E}-2 \pm 6.5 \mathrm{E}-05$ respectively for apo Can1. Cross-hairs mark the Guinier-Krakty point or peak position for an ideal, globular particle. b Paired-distance, $\mathrm{P}(\mathrm{r})$, -distribution function performed as an indirect Fourier transform using the Moore method. $P(r)$-distributions were normalised to particle's Porod volume using 121,421 $\AA^{3}$ and $132,291 \AA^{3}$ for the $\mathrm{Can} 1$ with $\mathrm{cA}_{4}$ (blue) and apo Can1 (black), respectively. Widening of the distribution for apo Can1 suggests larger distances are more accessible. c Fit of apo Can1 SAXS data (black circles) using atomistic model from constrained torsion angle molecular dynamics. Best single-state model (red line), $\chi^{2} 1.8$, identifies a model that opens through translation of the nuclease domain. The best open model was achieved where the CARF dimer was maintained as a rigid body and the nuclease-like domain was allowed to vary $\pm 5 \AA$ from its observed position. The nuclease domain was treated as a separate, rigid body tethered by an unconstrained loop (residues 423 to 428). Residuals of the fit are presented. The inset shows the fit of the Can 1

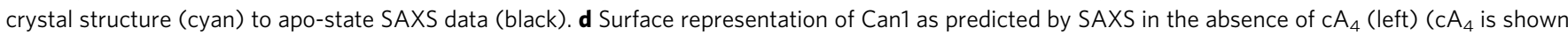
to illustrate the binding position and demonstrates the CARF domains are immobile) and the structure of Can1 in complex with cA $\mathrm{A}_{4}$ solved by $\mathrm{X}$-ray crystallography (right). Coloured as first CARF domain (magenta), nuclease-like domain (cyan), second CARF domain (green), nuclease domain (orange). Loops between the domains are not shown for clarity. A molecule of $\mathrm{CA}_{4}$ bound to $\mathrm{Can} 1$ is shown in yellow spheres. e The crystal structure of $\mathrm{Can} 1$ shown in cartoon form coloured by temperature factor from white (high temperature factor) to dark purple (low temperature factor). $\mathrm{A}_{\text {molecule of }} \mathrm{c} \mathrm{A}_{4}$ bound to Can1 is shown in yellow spheres.

VC1899 structures, we treated this unit as a rigid body. We generated $\sim 2500$ conformations that were subsequently fit to the apo state SAXS data. A best single-state model was found (best $\chi^{2}$ 1.81; Fig. 6c, d), where there is movement of the nuclease and nuclease-like domains away from the core CARF dimer. This has the effect of exposing the $\mathrm{CA}_{4}$ binding pocket to solvent, which is required for binding. Analysis of the temperature factor of residues in Can1 (Fig. 6e) shows the nuclease domain residues have higher average temperature factors than the other domains in Can1, which supports the hypothesis that the nuclease domain is more mobile. We envisage Can1 in solution is sampling between the open and closed states, exposing the $\mathrm{cA}_{4}$ binding site in the CARF dimer. Upon $\mathrm{CA}_{4}$ binding, the closed conformation of the protein is stabilised, as observed in the crystal structure. A potential 'hinge' between the second CARF domain and the nuclease domain to allow these open and closed states is the connecting loop which contains a Gly423/Arg424/Pro425 motif. The loop between the first CARF domain and the nuclease-like domain is longer, and contains two 'PPG' motifs (Pro160/Pro161/ Gly162 and Pro165/Pro166/Gly167) which are likely to be important in providing flexibility between the domains. A superimposition of Can1 onto VC1899, structurally aligned on the CARF domain dimer from each protein only, suggests the possibility of movement of the nuclease and nuclease-like domains between the open and closed states (Supplementary Fig. 10). It is possible that a complex population of open and closed structures exists in the absence of $\mathrm{cA}_{4}$.

\section{Discussion}

The structure of the Can1 enzyme clearly demonstrates that the two halves of the protein, comprising a CARF domain $\mathrm{N}$-terminal to a nuclease fold domain, are related to one another. Coupled to the observation that each half is clearly related to the DUF1887 family, as exemplified by the VC1889 protein, we can postulate a scenario whereby gene duplication, fusion and subsequent divergent evolution of an ancestral DUF1887 family member gave rise to the Can1 family. Can1 appears to be limited to members of the genus Thermus, but in contrast DUF1887 is much more widely distributed, with representatives found throughout the bacterial phyla and is particularly common in the proteobacteria. Although no member of the DUF1887 family has yet been characterised biochemically, given the relationship with Canl and the conservation of a nuclease active site we suggest the provisional name "Can2" (CRISPR ancillary nuclease 2) for this family. 
Analysis of the structure of a CARF domain protein bound to its cognate cyclic oligoadenylate ligand can be used to draw some general conclusions about the mechanism of recognition. Canl is unusual in being a monomeric protein with two non-identical CARF domains, and therefore possesses less intrinsic symmetry than most family members. $\mathrm{cA}_{4}$ is bound in a markedly asymmetrical conformation, which is in part exaggerated by the position of one adenine base in an axial position, driven by a stacking interaction with a tryptophan residue. Many different residues, the vast majority from the two CARF domains, contribute to the ligand binding pocket, which appears to be largely pre-formed in the apo protein. A significant number of these interactions are with protein main chain atoms, and although some of these are structurally conserved between the two 'halves' of Can1, the difference in side chain residue meant they would not have been predicted by sequence alone. Site-directed mutagenesis has highlighted a key role of Lys90, which occupies a central position in CARF domain 1 at the $\mathrm{N}$-terminus of an $\alpha$ helix, by forming a salt bridge with a bridging phosphate of $\mathrm{CA}_{4}$; basic residues at an equivalent position have been shown to be essential for $\mathrm{CA}_{4}$ recognition in other CARF family proteins ${ }^{10,12}$.

Recently, the structures of two CARF-HEPN family ribonucleases, Csm6 from Thermococcus onnurineus and Csx1 from S. islandicus, have been solved with $\mathrm{cA}_{4}$ bound ${ }^{31,32}$. Although each of these proteins have the basic unit of a CARF domain for $\mathrm{CA}_{4}$ binding in common with Canl, there is little sequence conservation across the three proteins. In particular, Can1 lacks conserved Y14, Y19, S51, H155, and $\mathrm{N} 158 \mathrm{cA}_{4}$-interacting residues identified as conserved across the Csx1/Csm6 family ${ }^{32}$. N158 is replaced by K90 in Can1, reflecting the importance of this position in the centre of the CARF domain for $\mathrm{cA}_{4}$ binding despite a lack of sequence conservation. Residues W14 and H132, identified as important for $\mathrm{cA}_{4}$ cleavage by $\mathrm{Csm}^{31}$, are also not conserved in Can1, and we see no evidence for ring nuclease activity by Can1 in vitro. The three enzymes bind $\mathrm{cA}_{4}$ in different orientations, emphasising the conformational flexibility possible for this large cyclic nucleotide.

The $\mathrm{CA}_{4}$ ligand is completely buried in the Can1 complex, demonstrating that significant protein conformational changes are required to facilitate binding. The close structural agreement observed for the two CARF domains of Can1 compared to the VC1899 apo protein suggests that the CARF dimer conformation does not change radically upon $\mathrm{CA}_{4}$ binding. In contrast, the temperature factors of the residues in the crystal structure, coupled with SAXS analysis, suggest that the nuclease domain is mobile, which would help facilitate $\mathrm{cA}_{4}$ binding to apo Can1. Modelling of dsDNA binding by the nuclease domain, coupled with the observation that conserved basic residues in the nuclease-like domain are essential for activity (presumably due to a role in DNA binding rather than directly in catalysis), suggests that $\mathrm{cA}_{4}$ binding allows the nuclease and nuclease-like domains to assemble into a functional dsDNA binding site, activating the enzyme. Modelling of dsDNA from homologous structures suggests a further modest closure of the cleft between the two domains may occur upon DNA binding, although this will depend on the precise position of the dsDNA substrate bound to Can1.

An emerging theme of CRISPR-based defence systems is the role of collateral (non-specific) nucleic acid degradation in immunity (reviewed in ref. ${ }^{33}$ ). In addition to the cOA activated, non-specific ribonucleases described above for type III systems, type VI (Cas13) effectors provide effective immunity against mobile genetic elements by degrading RNA non-specifically ${ }^{34,35}$. The mechanism for this immunity was recently shown to result in degradation of host, rather than virus, RNA, driving infected cells into dormancy and thus preventing the spread of infection ${ }^{36}$.
DNA is also a target for collateral degradation. Type III effectors hydrolyse ssDNA on binding target RNA - an activity that appears largely non-specific in vitro ${ }^{37-39}$, but which may favour transcribing phage DNA in vivo ${ }^{40}$. Likewise, type $\mathrm{V}$ effectors such as Cas12a possess a non-specific ssDNA degradation activity that is licensed by crRNA-dependent DNA binding ${ }^{41,42}$.

Here, we have described another option available to CRISPR defence systems: cyclic oligoadenylate-dependent supercoiled DNA cleavage by the Can1 enzyme. Once activated by $\mathrm{cA}_{4}$ generated in response to detection of foreign RNA, Can1 cleaves supercoiled DNA to generate nicked products. In vivo, such an activity is likely to slow down the replication kinetics of rapidlyreplicating phage, where nicks can result in the collapse of DNA replication forks to generate double-strand breaks. In contrast, such nicks are not too great a burden for the slowly-replicating host chromosome as they are easily repaired by DNA ligase. This nickase activity will operate in parallel with non-specific RNA degradation by the T. thermophilus Csm6 enzymes (Fig. 7), providing layered defence against mobile genetic elements detected by the CRISPR system. Given the structural conservation of CARF and nuclease domains in the Can2 (DUF1887) family, we predict that this family, which is widespread in bacteria, functions in a similar way to Can1 by cleaving supercoiled DNA. However, there is a key difference-the Can2 homodimer has two nuclease active sites, which may have functional consequences. In conclusion, we have revealed key molecular details of the cOAmediated anti-viral defence mediated by type III CRISPR systems -one of the most common types of CRISPR effectors found in nature.

\section{Methods}

Expression and purification of Can1 and variants. Two overlapping gBlock fragments to form the synthetic can 1 gene were purchased from Integrated DNA Technologies, Coralville, IA, United States (IDT) and cloned into the pEV5HisTEV vector using NcoI and HindIII restriction enzyme sites ${ }^{25}$. The cloning strategy resulted in the addition of two residues (Leu and Glu) to the C-terminus of the protein. The full sequence of the synthetic gene is shown in Supplementary Table 1. The variants E541A/D543A, K90E, N12A, W42A, H113A, Q222E, and R206E/ R249E were generated using the QuikChange Site-Directed Mutagenesis kit as per manufacturer's instructions (Agilent Technologies). Primer sequences for mutagenesis are shown in Supplementary Table 2. The pEV5HisTEV-can1 wild-type and variant constructs were transformed into C43 (DE3) E. coli cells (Sigma Aldrich). Protein expression was induced with $0.4 \mathrm{mM}$ isopropyl- $\beta$-D-1-thiogalactoside (IPTG) at an $\mathrm{OD}_{600}$ of $\sim 0.6$ and grown overnight at $25^{\circ} \mathrm{C}$. Cells were harvested and resuspended in lysis buffer containing $50 \mathrm{mM}$ Tris- $\mathrm{HCl} \mathrm{pH} \mathrm{7.5,500}$ $\mathrm{mM} \mathrm{NaCl}, 10 \mathrm{mM}$ imidazole and $10 \%$ glycerol, and lysed by sonicating six times for $2 \mathrm{~min}$ on ice with $2 \mathrm{~min}$ rest intervals. Can 1 was purified with a $5 \mathrm{ml}$ HisTrapFF column (GE Healthcare), washed with 5 column volumes (CV) of buffer containing $50 \mathrm{mM}$ Tris- $\mathrm{HCl} \mathrm{pH} 7.5,500 \mathrm{mM} \mathrm{NaCl}, 30 \mathrm{mM}$ imidazole and $10 \%$ glycerol, and eluted with a linear gradient of buffer containing $50 \mathrm{mM}$ Tris- $\mathrm{HCl} \mathrm{pH} 7.5,500 \mathrm{mM}$ $\mathrm{NaCl}, 500 \mathrm{mM}$ imidazole and $10 \%$ glycerol across $15 \mathrm{CV}$. Protein containing fractions were concentrated and the hexa-histidine affinity tag was removed by incubating protein with Tobacco Etch Virus (TEV) protease (10:1) overnight at room temperature.

Cleaved Can 1 was isolated from the TEV protease by repeating the immobilised metal affinity chromatography step and collecting the unbound fraction. Size exclusion chromatography was used to further purify Can1, eluting protein isocratically with buffer containing $20 \mathrm{mM}$ Tris- $\mathrm{HCl} \mathrm{pH} \mathrm{7.5,150} \mathrm{mM} \mathrm{NaCl}$. The protein was concentrated using a centrifugal concentrator, aliquoted and frozen at $-80^{\circ} \mathrm{C}$. For seleno-methionine labelling, the pEV5HisTEV-Canl construct was transformed into B834 (DE3) E. coli (Novagen) and cultures grown in M9 minimal medium supplemented with Selenomethionine Nutrient Mix (Molecular Dimensions, Newmarket, Suffolk, UK) and $50 \mathrm{mg} \mathrm{L}^{-1}$ (L)-selenomethionine (Acros Organics). Protein was purified as described above.

Can1 nuclease assay. In total $200 \mathrm{nM}$ Can1 was incubated with $2 \mathrm{nM}$ plasmid pEV5HisTEV for the time indicated at $60^{\circ} \mathrm{C}$ in $20 \mu \mathrm{l}$ final reaction volume in $20 \mathrm{mM}$ MES pH $6.5,100 \mathrm{mM} \mathrm{NaCl}$ and $1 \mathrm{mM}$ EDTA supplemented with $5 \mathrm{mM}$ $\mathrm{MnCl}_{2}$ and $200 \mathrm{nM} \mathrm{cA}_{4}$. Reactions were stopped by addition of $10 \mathrm{mM}$ EDTA. Control reactions were carried out by incubating plasmid without protein, $\mathrm{MnCl}_{2}$ or $\mathrm{CA}_{4}$ under the same conditions. For kinetic analysis, triplicate experiments were carried out by incubating Can 1 in $140 \mu \mathrm{l}$ final reaction volume under the conditions described above. $20 \mu \mathrm{l}$ was removed and quenched by addition of $2 \mu \mathrm{l}$ 


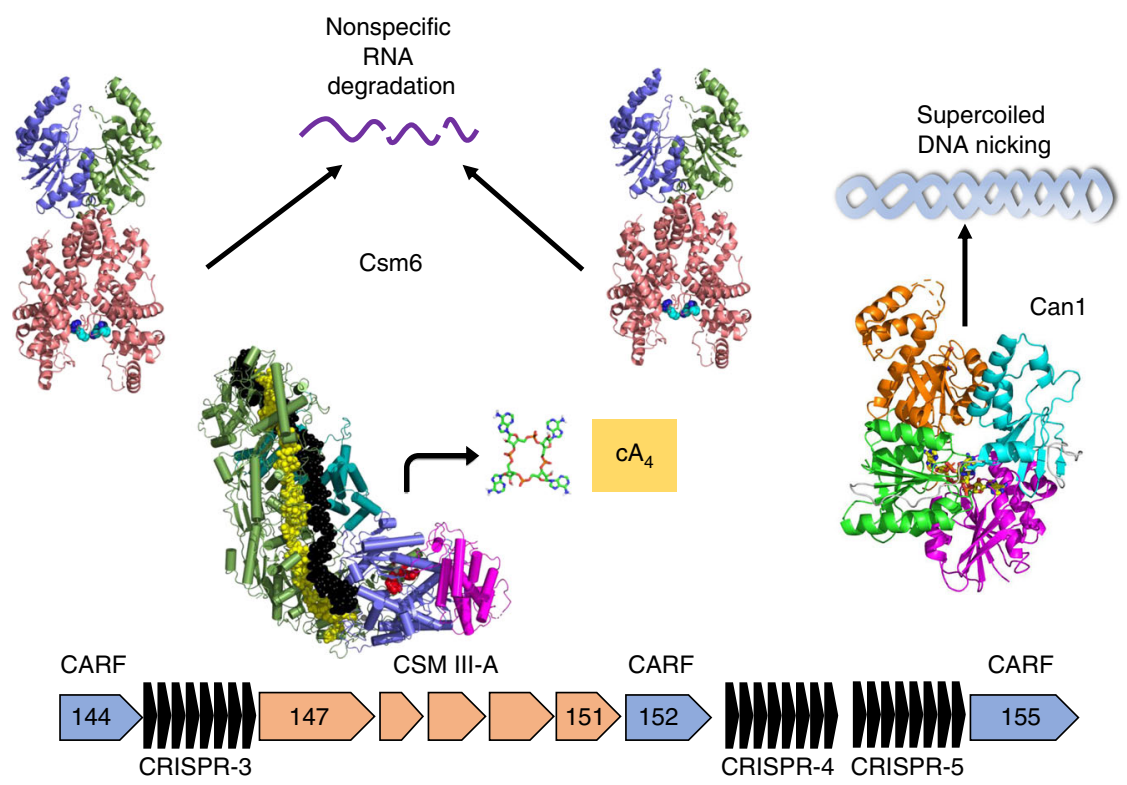

Fig. 7 Cartoon of the type III-A CRISPR defence system of T. thermophilus. The type III-A effector binds phage RNA, activating the cyclase domain which synthesises $\mathrm{CA}_{4}$ from ATP. cA 4 binds to and activates the two Csm6 family RNases (encoded by tthb144 and tthb152) and the DNA nickase Can1 (encoded by thb155), providing a multi-layered defence against mobile genetic elements.

$100 \mathrm{mM}$ EDTA at desired time points. To differentiate nuclease activity of wild type Can1 and all variants, the kinetic assay was changed by reducing protein and $\mathrm{cA}_{4}$ concentration to $100 \mathrm{nM}$ and $20 \mathrm{nM}$, respectively. After adding $4 \mu \mathrm{l} 6 \times$ DNA loading dye (New England BioLabs, Ipswich, MA, United States), $10 \mu \mathrm{l}$ sample was analysed by $0.7 \%$ native agarose gel electrophoresis. Gels were scanned by Typhoon FLA 7000 imager (GE Healthcare) at a wavelength of $532 \mathrm{~nm}$, quantified using the Bio-Formats plugin ${ }^{43}$ of ImageJ as distributed in the Fiji package ${ }^{44}$ and plotted against the time using Kaleidagraph (Synergy Software, Reading, PA, United States). The data were fitted to a single exponential curve ${ }^{1}$.

Nicking endonuclease digestion. Nicking endonuclease Nt.BspQI (New England BioLabs, Ipswich, MA, United States) hydrolyses the phosphodiester bond between the 3'-hydroxyl and 5'-phosphate on one strand of dsDNA. Therefore, the open circle conformation of pEV5HisTEV containing the unique GCTCTTCN^ ${ }^{\wedge}$ site was generated by Nt.BspQI. All incubations with commercial enzymes followed manufacturer's instructions.

Alkaline agarose gel electrophoresis. DNA products were separated on $0.7 \%$ alkaline agarose gels ( $30 \mathrm{mM} \mathrm{NaCl}, 2 \mathrm{mM}$ EDTA and $0.7 \%$ agarose). The gels were immersed for $2 \mathrm{~h}$ in alkaline electrophoresis buffer $(30 \mathrm{mM} \mathrm{NaOH}, 2 \mathrm{mM}$ EDTA) before running. Samples and ladder were denatured with $6 \times$ alkaline electrophoresis loading dye ( $180 \mathrm{mM} \mathrm{NaOH}, 6 \mathrm{mM}$ EDTA, $18 \%$ Ficoll 400 and $0.05 \%$ bromophenol blue) and heated at $95^{\circ} \mathrm{C}$ for $5 \mathrm{~min}$, and then chilled on ice. Gels were run at $2 \mathrm{~V} \mathrm{~cm}^{-1}$ for $10 \mathrm{~h}$ in alkaline electrophoresis buffer, then soaked in renaturing buffer ( $500 \mathrm{mM}$ Tris- $\mathrm{HCl} \mathrm{pH} \mathrm{8.0)} \mathrm{for} 30 \mathrm{~min}$ and stained with SYBR Gold (Thermo Scientific, Waltham, MA, United States) for $30 \mathrm{~min}$. Gels were destained with $\mathrm{H}_{2} \mathrm{O}$ and scanned by Typhoon FLA 7000 imager at a wavelength of $532 \mathrm{~nm}$.

Plasmid ligation and supercoiling. In total $200 \mathrm{nM}$ Can1 was incubated with $2 \mathrm{nM}$ plasmid pEV5HisTEV in $20 \mu \mathrm{l}$ final reaction volume as described above. Reactions were quenched and deproteinized by PCR Clean-Up System (Promega, Madison, Wisconsin, United States). In total $10 \mu$ l eluted product was incubated with T4 DNA Ligase (New England BioLabs, Ipswich, MA, United States) in ligase buffer (50 mM Tris-HCl pH 7.5, $10 \mathrm{mM} \mathrm{MgCl}, 1 \mathrm{mM}$ ATP and $10 \mathrm{mM}$ DTT) in $20 \mu \mathrm{l}$ final reaction volume following manufacturer's instructions. After inactivating at $65^{\circ} \mathrm{C}$ for $10 \mathrm{~min}$, ligated products were incubated with E. coli DNA gyrase (Inspiralis, Norwich, UK) in gyrase buffer $(35 \mathrm{mM}$ Tris- $\mathrm{HCl} \mathrm{pH} \mathrm{7.5,} 24 \mathrm{mM} \mathrm{KCl}$, $4 \mathrm{mM} \mathrm{MgCl} 2,2 \mathrm{mM}$ DTT, $1.8 \mathrm{mM}$ spermidine, $1 \mathrm{mM}$ ATP, $6.5 \%$ glycerol and $0.1 \mathrm{mg} \mathrm{mL}^{-1}$ albumin) in $40 \mu \mathrm{l}$ final reaction volume as per manufacturer's instructions. Reactions were deproteinized by phenol chloroform extraction before analysis by agarose electrophoresis.

Preparation of $\mathbf{c A}_{\mathbf{4}} \cdot \mathrm{cA}_{4}$ was purchased from Biolog Life Science Institute, Bremen, Germany. ${ }^{32} \mathrm{P}_{\text {labelled }} \mathrm{cA}_{4}$ was prepared by incubating $120 \mu \mathrm{g}$ S. solfataricus Csm complex with $0.5 \mathrm{mM}$ ATP, $1 \mathrm{mM} \mathrm{MgCl}$ and $100 \mathrm{nM}$ target RNA in $20 \mathrm{mM}$ MES pH 5.5, $100 \mathrm{mM}$ K-glutamate and $1 \mathrm{mM}$ DTT for $2 \mathrm{~h}$ at $70^{\circ} \mathrm{C}$. The reaction product was isolated by phenol-chloroform extraction, followed by chloroform extraction, and frozen at $-20^{\circ} \mathrm{C}^{25}$.

Can1 and $\mathbf{c A}_{\mathbf{4}}$ electrophoretic mobility shift assay. In total $100 \mathrm{nM}{ }^{32} \mathrm{P}$ labelled $\mathrm{cA}_{4}$ was incubated with different concentrations of Can1 for $10 \mathrm{~min}$ at room temperature in $10 \mu \mathrm{l}$ final reaction volume in $20 \mathrm{mM}$ MES pH $6.5,100 \mathrm{mM} \mathrm{NaCl}$, $1 \mathrm{mM}$ EDTA, $5 \mathrm{mM} \mathrm{MnCl}$ and $2 \mu \mathrm{M}$ BSA. In total $10 \mu \mathrm{l}$ of $20 \%$ glycerol was added to each reaction before loading onto native acrylamide gel $(15 \%$ acrylamide, $1 \times$ TBE). ${ }^{32} \mathrm{P}$ labelled $\mathrm{CA}_{4}$ was visualised by phosphorimaging using a Typhoon FLA 7000 imager (GE Healthcare). All images were analysed by Bio-Formats plugin ${ }^{43}$ of ImageJ as distributed in the Fiji package ${ }^{44}$.

Crystallisation of Can1. Selenomethionine labelled Can1 at $10.4 \mathrm{mg} \mathrm{mL}^{-1}$ was mixed in a 1:2 molar ratio with $\mathrm{cA}_{4}$ and incubated at room temperature for $30 \mathrm{~min}$, before centrifugation at $13,000 \mathrm{rpm}$ prior to crystallisation. Sitting drop vapour diffusion experiments were set up at the nanoliter scale using both commercially available and in-house crystallisation screens, and subsequently incubated at $293 \mathrm{~K}$. Crystals appeared in many conditions, but those used for data collection grew from a reservoir solution of $20 \%$ PEG 3350, $200 \mathrm{mM}$ sodium citrate, and $100 \mathrm{mM}$ bisTris propane, $\mathrm{pH}$ 6.5. Crystals were harvested and transferred to a drop of reservoir solution with the addition of $20 \%$ glycerol before cryo-cooling. Attempts to crystallise Canl in the absence of $\mathrm{cA}_{4}$ did not yield crystals.

X-ray data processing, structure solution, and refinement. X-ray data were collected at a wavelength of $0.9159 \AA$, on beamline I04-1 at Diamond Light Source, at $100 \mathrm{~K}$ to $1.83 \AA$ $\AA$ resolution. Data were automatically processed using Xia $2^{45}$ using XDS and XSCALE ${ }^{46}$. A strong anomalous signal was detected to $2.36 \AA$ resolution. The data were phased using AutoSol in Phenix and the initial model was built in AutoBuild ${ }^{47}$. Model refinement was achieved by iterative cycles of REFMAC5 $5^{48}$ in the CCP4 suite ${ }^{49}$ and manual manipulation in $\mathrm{COOT}^{50}$. Electron density for $\mathrm{CA}_{4}$ was clearly visible in the maximum likelihood $/ \sigma_{A}$ weighted $F_{\text {obs }}-F_{\text {calc }}$ electron density map at $3 \sigma$. The coordinates for $\mathrm{cA}_{4}$ were generated in ChemDraw (Perkin Elmer) and the library was generated using acedrg ${ }^{27}$, before fitting of the molecule in COOT. Model quality was monitored throughout using Molprobity ${ }^{51}$ (score 1.05; centile 100). Ramachandran statistics are $98.5 \%$ favoured, $0 \%$ disallowed. Data and refinement statistics are shown in Supplementary Table 3. The coordinates and data have been deposited in the Protein Data Bank with deposition code 6SCE.

Small angle X-ray scattering. The apo-state of Can1 was measured using size exclusion chromatography coupled to small angle X-ray (SEC-SAXS) on beamline B21 at Diamond Light Source. A $4.8 \mathrm{ml}$ Shodex KW- 402.5 column was preequilibrated with 3 column volumes of buffer containing $20 \mathrm{mM}$ Tris $\mathrm{pH} 7.5$ and $150 \mathrm{mM} \mathrm{NaCl}$. SEC-SAXS experiments were measured at a flow rate of $0.160 \mathrm{ml}$ per minute with $2 \mathrm{~s}$ exposure per frame in a $1.5 \mathrm{~mm}$ diameter, $10 \mu \mathrm{m}$ thick quartz capillary flow cell and a $800 \mu \mathrm{m}$ squared X-ray beam focused on an Eiger $4 \mathrm{M}$ detector $4 \mathrm{~m}$ from the sample. Column performance and instrumentation 
calibration was checked by measuring a $45 \mu \mathrm{l}$ sample of BSA injected at $10 \mathrm{mg} \mathrm{mL}$ ${ }^{-1}$. In total $45 \mu \mathrm{l}$ of the apo-state of Can1 was injected $\left(10.4 \mathrm{mg} \mathrm{mL}^{-1}, 140 \mu \mathrm{M}\right)$ and frames were collected for a total of $30 \mathrm{~min}$. The flat radius-of-gyration, $R_{\mathrm{g}}$, and nearly ideal overlay of the frames constituting the merged frames suggests the measured frames are free of interparticle interference (Supplementary Data 1).

To measure Can1 in the presence of $\mathrm{CA}_{4}$, a SEC purification of Can1 was performed at the beamline by manually collecting the peak fraction $(\sim 100 \mu \mathrm{l})$ during an additional SEC run of the same $10.4 \mathrm{mg} \mathrm{mL}^{-1}$ protein stock. The peak fraction represented a dilution of $\sim 3.2 \times$ for a final concentration of $44 \mu \mathrm{M}$ of purified protein. A corresponding buffer blank $(\sim 1 \mathrm{ml})$ was collected before sample injection. Samples were mixed with ligand to a final concentration of $0.25 \mathrm{mM}$ and 3 dilutions of the sample were made first by making a two-thirds dilution of the master stock followed by two consecutive nine-tenths dilutions. Dilutions were performed with the buffer blank supplemented with ligand to maintain a ratio of 3to-1 ligand-to-protein ratio. Samples were processed in batching mode at $25 \mu \mathrm{l}$ per sample using the Arinax sample handling robot.

Raw SAXS images were processed with the DAWN $\mathrm{N}^{52}$ processing pipeline at the beamline to produce normalised, integrated 1-D un-subtracted SAXS curves. SECSAXS analysis and buffer subtractions were performed with the program ScÅtter (www.bioisis.net). Modelling of the apo-state was performed using the program CNS version 1.3 (cns-online.org/v1.3) ${ }^{53}$. First, missing loops and tails from the Xray crystal structure were added by building an extended chain model of Can1 with generate_extended.inp. Protein only distance restraints were extracted from the ligand bound crystal structure of Can1 and used as pseudo-NOE constraints to fold the extended chain into the known parts of the crystal structure (model_anneal. inp). The resulting structure matched the crystal structure with missing loops and tails added back but in unconstrained positions. The folded structure (groundstate) was used as a starting conformation for limited rounds of simulated annealing or constant temperature simulations (anneal.inp) to sample various conformations as domain-to-domain distance restraints were varied. Specifically, the two CARF domains were kept as a rigid unit, whereas either the nuclease or nuclease-like domains were allowed to vary from their ground-state positions as rigid bodies. An initial simulation allowed the non-CARF domains to move as unconstrained rigid bodies only tethered by the linking peptide regions. Additional simulations were performed that constrained the non-CARF domains within 5 and $10 \AA ̊$ of their observed crystallographic positions. These were loose restraints added between the centre-of-masses for each domain based on the $\mathrm{P}(\mathrm{r})$-distribution of the apo-state SAXS data. Specifically, no distances were allowed to exceed $d_{\max }$ and distances were given upper and lower limits determined as $\pi / q_{\max }$. A total of 2430 conformations were generated and used as an ensemble fit with the online web-app multi-FOXS ${ }^{54}$ which identified the best two-state model for the apo-state. Single model fits were performed with FOXS (http://modbase.compbio.ucsf.edu/foxs) ${ }^{55}$

Reporting summary. Further information on research design is available in the Nature Research Reporting Summary linked to this article.

\section{Data availability}

The final protein model and structure factors presented in this study have been deposited in the Protein DataBank with the accession code 6SCE. The SAXS data has been deposited in bioisis with the accession codes CAN1AP and CAN1C4. The source data underlying Figs. 4b-d, 5a, b and Supplementary Fig. 5a, b are provided as a Source Data file. Other data presented in this study are available from the corresponding authors upon reasonable request.

Received: 18 July 2019; Accepted: 13 December 2019; Published online: 24 January 2020

\section{References}

1. Rouillon, C., Athukoralage, J. S., Graham, S., Grüschow, S. \& White, M. F. Control of cyclic oligoadenylate synthesis in a type III CRISPR system. eLife 7, e36734 (2018).

2. Niewoehner, O. et al. Type III CRISPR-Cas systems produce cyclic oligoadenylate second messengers. Nature 548, 543-548 (2017).

3. Kazlauskiene, M., Kostiuk, G., Venclovas, C., Tamulaitis, G. \& Siksnys, V. A cyclic oligonucleotide signaling pathway in type III CRISPR-Cas systems. Science 357, 605-609 (2017).

4. Nasef, M. et al. Regulation of cyclic oligoadenylate synthesis by the $S$. epidermidis Cas10-Csm complex. RNA 25, 948-962 (2019).

5. Koonin, E. V. \& Makarova, K. S. Discovery of oligonucleotide signaling mediated by CRISPR-associated polymerases solves two puzzles but leaves an enigma. ACS Chem. Biol. 13, 309-312 (2018).

6. Niewoehner, O. \& Jinek, M. Structural basis for the endoribonuclease activity of the type III-A CRISPR-associated protein Csm6. RNA 22, 318-329 (2016).
7. Foster, K., Kalter, J., Woodside, W., Terns, R. M. \& Terns, M. P. The ribonuclease activity of Csm6 is required for anti-plasmid immunity by Type III-A CRISPR-Cas systems. RNA Biol. 16, 449-460 (2019).

8. Sheppard, N. F., Glover, C. V. 3rd, Terns, R. M. \& Terns, M. P. The CRISPRassociated Csx1 protein of Pyrococcus furiosus is an adenosine-specific endoribonuclease. RNA 22, 216-224 (2016).

9. Han, W., Pan, S., Lopez-Mendez, B., Montoya, G. \& She, Q. Allosteric regulation of Csx1, a type IIIB-associated CARF domain ribonuclease by RNAs carrying a tetraadenylate tail. Nucleic Acids Res. 45, 10740-10750 (2017).

10. Athukoralage, J. S., Graham, S., Grüschow, S., Rouillon, C. \& White, M. F. A type III CRISPR ancillary ribonuclease degrades its cyclic oligoadenylate activator. J. Mol. Biol. 431, 2894-2899 (2019).

11. Lintner, N. G. et al. The structure of the CRISPR-associated protein Csa3 provides insight into the regulation of the CRISPR/Cas system. J. Mol. Biol. 405, 939-955 (2011).

12. Athukoralage, J. S., Rouillon, C., Graham, S., Grüschow, S. \& White, M. F. Ring nucleases deactivate Type III CRISPR ribonucleases by degrading cyclic oligoadenylate. Nature 562, 277-280 (2018).

13. Makarova, K. S., Anantharaman, V., Grishin, N. V., Koonin, E. V. \& Aravind, L. CARF and WYL domains: ligand-binding regulators of prokaryotic defense systems. Front Genet. 5, 102 (2014).

14. Deng, L., Garrett, R. A., Shah, S. A., Peng, X. \& She, Q. A novel interference mechanism by a type IIIB CRISPR-Cmr module in Sulfolobus. Mol. Microbiol 87, 1088-1099 (2013).

15. Hatoum-Aslan, A., Maniv, I., Samai, P. \& Marraffini, L. A. Genetic characterization of antiplasmid immunity through a Type III-A CRISPR-Cas system. J. Bacteriol. 196, 310-317 (2014).

16. Jiang, W., Samai, P. \& Marraffini, L. A. Degradation of phage transcripts by CRISPR-associated rnases enables Type III CRISPR-Cas immunity. Cell 164, 710-721 (2016)

17. Rostol, J. T. \& Marraffini, L. A. Non-specific degradation of transcripts promotes plasmid clearance during type III-A CRISPR-Cas immunity. Nat. Microbiol 4, 656-662 (2019).

18. Staals, R. H. et al. RNA targeting by the type III-A CRISPR-Cas Csm complex of Thermus thermophilus. Mol. Cell 56, 518-530 (2014).

19. Staals, R. H. et al. Structure and activity of the RNA-targeting Type III-B CRISPR-Cas complex of Thermus thermophilus. Mol. Cell 52, 135-145 (2013).

20. Taylor, D. W. et al. Structural biology. Structures of the CRISPR-Cmr complex reveal mode of RNA target positioning. Science 348, 581-585 (2015).

21. Liu, T. Y., Iavarone, A. T. \& Doudna, J. A. RNA and DNA targeting by a reconstituted Thermus thermophilus Type III-A CRISPR-Cas system. PLoS ONE 12, e0170552 (2017).

22. Shah, S. A. et al. Comprehensive search for accessory proteins encoded with archaeal and bacterial type III CRISPR-cas gene cassettes reveals 39 new cas gene families. RNA Biol. 16, 530-542 (2019).

23. Agari, Y. et al. Transcription profile of Thermus thermophilus CRISPR systems after phage infection. J. Mol. Biol. 395, 270-281 (2010).

24. Zimmermann, L. et al. A completely reimplemented MPI bioinformatics toolkit with a New HHpred server at its core. J. Mol. Biol. 430, 2237-2243 (2018).

25. Rouillon, C., Athukoralage, J. S., Graham, S., Grüschow, S. \& White, M. F. Investigation of the cyclic oligoadenylate signalling pathway of type III CRISPR systems. Methods Enzymol. 616, 191-218 (2019).

26. Yang, W. Nucleases: diversity of structure, function and mechanism. Q Rev. Biophys. 44, 1-93 (2011).

27. Long, F. et al. AceDRG: a stereochemical description generator for ligands. Acta Crystallogr D. Struct. Biol. 73, 112-122 (2017).

28. Holm, L. \& Laakso, L. M. Dali server update. Nucleic Acids Res. 44, W351-W355 (2016).

29. Tamulaitiene, G. et al. Restriction endonuclease AgeI is a monomer which dimerizes to cleave DNA. Nucleic Acids Res. 45, 3547-3558 (2017).

30. Deibert, M., Grazulis, S., Sasnauskas, G., Siksnys, V. \& Huber, R. Structure of the tetrameric restriction endonuclease NgoMIV in complex with cleaved DNA. Nat. Struct. Biol. 7, 792-799 (2000).

31. Jia, N., Jones, R., Yang, G., Ouerfelli, O. \& Patel, D. J. CRISPR-Cas III-A Csm6 CARF domain is a ring nuclease triggering stepwise cA4 Cleavage with ApA $>$ p formation terminating RNase activity. Mol. Cell 75, 944-956 e6 (2019).

32. Molina, R. et al. Structure of Csx1-cOA4 complex reveals the basis of RNA decay in Type III-B CRISPR-Cas. Nat. Commun. 10, 4302 (2019).

33. Varble, A. \& Marraffini, L. A. Three new Cs for CRISPR: collateral, communicate, cooperate. Trends Genet 35, 446-456 (2019).

34. Abudayyeh, O. O. et al. C2c2 is a single-component programmable RNAguided RNA-targeting CRISPR effector. Science 353, aaf5573 (2016).

35. Meeske, A. J. \& Marraffini, L. A. RNA guide complementarity prevents selftargeting in Type VI CRISPR systems. Mol. Cell 71, 791-801 e3 (2018). 
36. Meeske, A. J., Nakandakari-Higa, S. \& Marraffini, L. A. Cas13-induced cellular dormancy prevents the rise of CRISPR-resistant bacteriophage. Nature 570, 241-245 (2019).

37. Elmore, J. R. et al. Bipartite recognition of target RNAs activates DNA cleavage by the Type III-B CRISPR-Cas system. Genes Dev. 30, 447-459 (2016).

38. Estrella, M. A., Kuo, F. T., Bailey, S. \& RNA-activated, D. N. A. cleavage by the Type III-B CRISPR-Cas effector complex. Genes Dev. 30, 460-470 (2016).

39. Kazlauskiene, M., Tamulaitis, G., Kostiuk, G., Venclovas, C. \& Siksnys, V. Spatiotemporal control of Type III-A CRISPR-Cas immunity: coupling DNA degradation with the target RNA recognition. Mol. Cell 62, 295-306 (2016).

40. Samai, P. et al. Co-transcriptional DNA and RNA Cleavage during Type III CRISPR-Cas Immunity. Cell 161, 1164-1174 (2015).

41. Chen, J. S. et al. CRISPR-Cas12a target binding unleashes indiscriminate single-stranded DNase activity. Science 360, 436-439 (2018).

42. Li, S. Y. et al. CRISPR-Cas12a has both cis- and trans-cleavage activities on single-stranded DNA. Cell Res. 28, 491-493 (2018).

43. Linkert, M. et al. Metadata matters: access to image data in the real world. $J$. Cell Biol. 189, 777-782 (2010).

44. Schindelin, J. et al. Fiji: an open-source platform for biological-image analysis. Nat. Methods 9, 676-682 (2012).

45. Winter, G. xia2: an expert system for macromolecular crystallography data reduction. J. Appl Crystallogr 43, 186-190 (2010).

46. Kabsch, W. Xds. Integration, scaling, space-group assignment and postrefinement. Acta Crystallogr. D Biol. Crystallogr. 66, 125-132 (2010).

47. Adams, P. D. et al. PHENIX: a comprehensive Python-based system for macromolecular structure solution. Acta Crystallogr. D Biol Crystallogr. 66, 213-221 (2010).

48. Murshudov, G. N., Vagin, A. A. \& Dodson, E. J. Refinement of macromolecular structures by the maximum-likelihood method. Acta Crystallogr. D Biol. Crystallogr. 53, 240-255 (1997).

49. Winn, M. D. et al. Overview of the CCP4 suite and current developments. Acta Crystallogr. D Biol. Crystallogr. 67, 235-242 (2011).

50. Emsley, P., Lohkamp, B., Scott, W. G. \& Cowtan, K. Features and development of Coot. Acta Crystallogr. D Biol. Crystallogr. 66, 486-501 (2010).

51. Chen, V. B. et al. MolProbity: all-atom structure validation for macromolecular crystallography. Acta Crystallogr. D Biol. Crystallogr. 66, 12-21 (2010).

52. Basham, M. et al. Data Analysis WorkbeNch (DAWN). J. Synchrotron Radiat. 22, 853-858 (2015).

53. Brunger, A. T. Version 1.2 of the Crystallography and NMR system. Nat. Protoc. 2, 2728-2733 (2007).

54. Schneidman-Duhovny, D., Hammel, M., Tainer, J. A. \& Sali, A. FoXS, FoXSDock and MultiFoXS: Single-state and multi-state structural modeling of proteins and their complexes based on SAXS profiles. Nucleic Acids Res. 44, W424-W429 (2016).

55. Schneidman-Duhovny, D., Hammel, M., Tainer, J. A. \& Sali, A. Accurate SAXS profile computation and its assessment by contrast variation experiments. Biophys. J. 105, 962-974 (2013)

\section{Acknowledgements}

We are grateful to the University of St Andrews Mass Spectrometry facility, and to staff at Diamond Light Source (beamlines I04-1 and B21). This work was supported by grants from the Biotechnology and Biological Sciences Research Council (REF: BB/S000313/1 to M.F.W. and REF: BB/R008035/1 to T.M.G.) and the China Scholarship Council (REF: 201703780015 to W.Z.)

\section{Author contributions}

M.F.W., T.M.G., and R.R. planned the experiments. S.A.M. performed the crystallisation and X-ray crystallography work, W.Z. performed the biochemical assays, W.Z. and S.G. performed the gene cloning and protein expression, and R.R. performed the SAXS and MD studies. M.F.W., T.M.G., and R.R. analysed the data and wrote the paper, with input from all authors.

\section{Competing interests}

The authors declare no competing interests.

\section{Additional information}

Supplementary information is available for this paper at https://doi.org/10.1038/s41467 019-14222-x.

Correspondence and requests for materials should be addressed to M.F.W. or T.M.G.

Peer review information Nature Communications thanks Hong Li, Timothy M. Ryan and the other, anonymous, reviewer(s) for their contribution to the peer review of this work. Peer reviewer reports are available.

Reprints and permission information is available at http://www.nature.com/reprints

Publisher's note Springer Nature remains neutral with regard to jurisdictional claims in published maps and institutional affiliations.

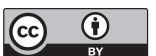

Open Access This article is licensed under a Creative Commons Attribution 4.0 International License, which permits use, sharing, adaptation, distribution and reproduction in any medium or format, as long as you give appropriate credit to the original author(s) and the source, provide a link to the Creative Commons license, and indicate if changes were made. The images or other third party material in this article are included in the article's Creative Commons license, unless indicated otherwise in a credit line to the material. If material is not included in the article's Creative Commons license and your intended use is not permitted by statutory regulation or exceeds the permitted use, you will need to obtain permission directly from the copyright holder. To view a copy of this license, visit http://creativecommons.org/ licenses/by/4.0/.

(C) The Author(s) 2020 